\title{
Examination of vesicular stomatitis virus-induced morphology changes in individual Vero cells by QMod microscopy
}

\author{
Isabel Scholz*,1, Christopher Montoya' \& Eric Vela ${ }^{1}$
}

\begin{abstract}
Viral infection of cultured cells induces changes in the biophysical characteristics of the affected cells. Advanced microscopic cameras such as Ovizio's QMod, coupled with the appropriate software, can measure a variety of characteristics on a per-cell basis. We have employed this system to monitor the progression of vesicular stomatitis virus infection in Vero cells and to describe the cellular changes associated with advancing vesicular stomatitis virus infection. The measurements of cellular characteristics are operator-independent, and the goal is to establish a robust method to mathematically determine viral infection levels in a given sample. This will provide a means to measure viral titer in a faster and less subjective way than manual reading of plaque assays or tissue culture infectious dose 50 assays.
\end{abstract}

\section{LAY ABSTRACT}

We describe changes in cellular characteristics at very early time points after viral infection. These measurements can be performed in a noninvasive manner, reducing hands-on operator time, assay variability and production of potentially hazardous materials. Infection can be monitored in the same sample over a period of time.

\section{METHOD SUMMARY}

Vero cells are imaged with a QMod microscope camera and analyzed in OsOne software. Features measured on individual cells are used to describe cellular morphology changes in response to infection with vesicular stomatitis virus expressing the Lassa virus Josiah glycoprotein (VSV $\Delta$ G/LASVGP). Characteristics of infection can be measured at very early time points after infection.

\section{KEYWORDS}

microscopy $\cdot$ Vero cells $\cdot$ viral vector $\cdot$ vesicular stomatitis virus

'Process Development, Ology Bioservices, Alachua, FL 32615, USA; *Author for correspondence: Isabel. scholz@ologybio.com

BioTechniques 68: 305-310 (June 2020) 10.2144/ btn-2019-0137

\section{GRAPHICAL ABSTRACT}

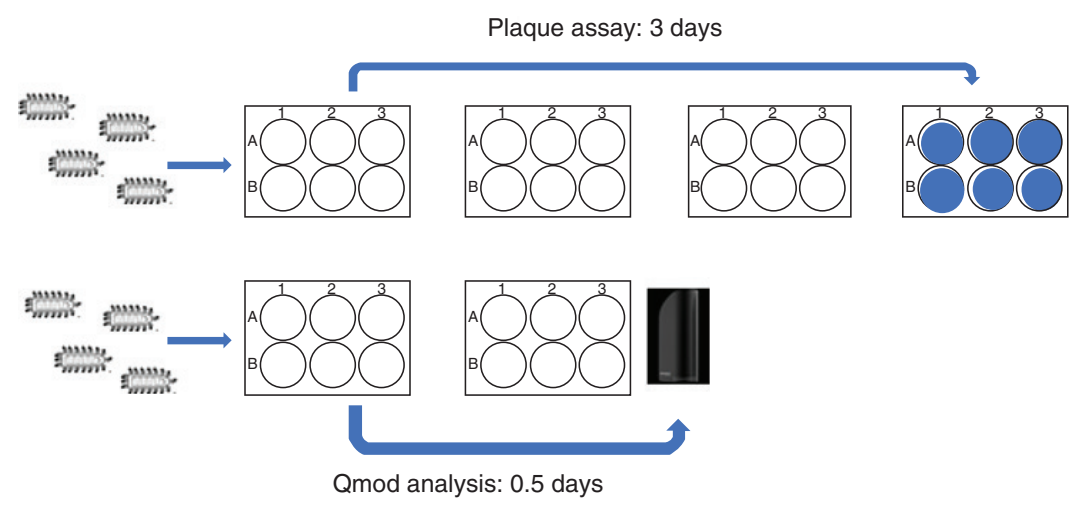

Time savings offered by QMod analysis of infected Vero cells.

Viral vectors are increasingly gaining importance in vaccine development, gene therapy and as oncolytic vectors. Vesicular stomatitis virus (VSV), an enveloped virus carrying a negative-sense RNA genome, has proven to be an excellent vaccine vector candidate against infectious diseases and specific cancers [1-3]. VSV has been shown to induce apoptosis in a variety of immortalized or tumorigenic cell types [4], and this capability is being exploited to construct oncolytic vectors $[5,6]$. Attenuated forms of VSV have been used in clinical trials and seroprevalence in humans remains low [7]. The viral RNA does not integrate into host cell genomes; and owing to the structural characteristics of the VSV particle, large foreign transgenes can be packaged and expressed within a VSV-pseudotyped virus expressing the various viral glycoproteins. VSV expressing glycoproteins from severely pathogenic viruses such as the Ebola [1], Lassa [8], Marburg [9], Nipah [10] and Zika virus [11] have been produced and tested in various models, and are capable of eliciting protective immunity at very high levels.
Viral vector manufacturing and process development rely on infectivity measurements for virus products. The plaque assay remains a mainstay of virology analytics. Plaques form in adherent cell monolayers, allowing for quantitation of plaque-forming units in the inoculum.

A variety of assays are available to determine yield of physical particles as well as infectious particles. However, several of the most well-characterized methods, such as plaque assays and tissue culture (TC) infectious dose 50 $\left(\mathrm{TCID}_{50}\right)$ assays, are associated with a wide variance and are also time- and labor-intensive. Thus, rapid and reliable means of assaying virus products are welcome advances, especially if the assay easily adapts to multiple product lines. Infected cells in adherent systems change markedly in their morphology, from elongated to rounded. This visible effect can be used to examine infectivity of virus samples. Ding et al. have studied the changes of 3T3 cells in response to VSV infection [12], and increasingly, single-cell analyses are being employed to examine the responses of cells to 
Table 1. Analyzed populations.

\begin{tabular}{|l|l|l|}
\hline Condition & \multicolumn{1}{|l|}{ Time postinfection (h) } & Cells analyzed (n) \\
\hline Mock & 2 & 2203 \\
\hline Mock & 4 & 2525 \\
\hline Mock & 6 & 1239 \\
\hline Mock & 8 & 2427 \\
\hline Mock & 10 & 2778 \\
\hline Infected & 2 & 2069 \\
\hline Infected & 4 & 2224 \\
\hline Infected & 6 & 2358 \\
\hline Infected & 8 & 2352 \\
\hline Infected & 10 & 2488 \\
\hline
\end{tabular}

Table 2. Titer results from each time point.

Time postinfection (h)

2

4

6

8

10

PFU: Plaque-forming unit.

viral infections. Additionally, Hebert et al. developed an elegant methodology (laser force cytology) to quantify levels of VSV infection in Vero cells [13]. This method analyzes cells based on their intrinsic properties, and they have established a metric to determine levels of infection in cell populations based on measurements obtained by laser force cytology using the LumaCyte (VA, USA) Radiance instrument. Yakimovich et al. examined cytopathic effects induced by Vaccinia virus, Herpes simplex virus and Rhinovirus by digital holotomographic microscopy and used refractive index gradient measurements of live cells to describe virus type-specific cytopathic effects. Their technique permitted label-free, noninvasive observations of virus-infected cells [14].

Ovizio Imaging Systems (Brussels, Belgium) has developed a line of microscopes for a variety of live-cell imaging applications for the biologics manufacturing industry. Its microscopy cameras and complementary software, OsOne, allow monitoring of viral infection kinetics in culture without the need for transferring or labeling cells. Digital holographic microscopy constructs a 3D hologram of the object of interest [15]. This technique allows for exami-
Titer (PFU/ml)

$5.0 \times 10^{3}$

$6.3 \times 10^{3}$

$2.3 \times 10^{3}$

Above range of dilutions plated

Above range of dilutions plated

nation and measurement of fine details of individual cells. Infection levels can be examined very early in the time course of infection, as measured by small changes in cellular morphology, changes that are not distinguishable by manual examination. The data obtained can be used for very fast and reproducible analysis of virus samples, thus lending important support to process decisions in viral vector manufacturing and process development.

In this report, we describe a novel viral infection analysis method using a live VSV-based Lassa virus (LASV) vaccine candidate. The recombinant VSV has been genetically altered to express the LASV Josiah glycoprotein (VSV $\Delta$ G/LASVGP), and infection in Vero cells was examined by microscopy using the Ovizio QMod camera and OsOne software. We assessed characteristics of infected Vero cells over the course of infection, obtained feature measurements of individual cells and examined very early biophysical distinctions of infected cells.

\section{MATERIALS \& METHODS}

\section{Cells \& viruses}

Vero cells (Ology Bioservices, Inc., FL, USA) were propagated in serum-free TC medium (proprietary formulation) on either CellBind or
TC-treated flatware (Corning, NY, USA) at $37^{\circ} \mathrm{C}$ and $7.5 \% \mathrm{CO}_{2}$. Cells were passaged to reach $80-95 \%$ confluence at the time of infection. The VSV $\Delta G / L A S V G P$ used for all experiments was graciously provided by the NIH National Institute of Allergy and Infectious Diseases under Material Transfer Agreement LAB-18-P_ LV-22 for in vitro use only and for training and research purposes only.

\section{Plaque assays}

Viral titers were determined by a standard plaque assay. Serial tenfold dilutions of virus in TC medium were prepared. Vero cells at 85-95\% confluence in six-well plates were infected with $300 \mu \mathrm{l}$ of inoculum per well for $1 \mathrm{~h}$ and rocked every 15 min during the $1-\mathrm{h}$ incubation. Following adsorption, inoculum was not removed, $3 \mathrm{ml}$ of overlays containing TC medium and $1 \%$ methylcellulose were placed on to the monolayers, and plates were incubated at $37^{\circ} \mathrm{C}$ and $7.5 \% \mathrm{CO}_{2}$ for 3 days. After incubation, overlays were removed, samples were stained with Crystal Violet ( $0.8 \%$ in methanol) for 30 min, washed twoto three-times with PBS without calcium or magnesium (Gibco, MA, USA), and plaques were manually counted using a light box.

\section{Microscopy}

Microscopy was performed using a Nikon Ts2R-FL microscope (Nikon Instruments, NY, USA). Analysis and 3D imaging were performed with a QMod camera and OsOne software (Ovizio Systems). Images were taken at $4 \times$ and $10 \times$ magnifications using Nikon Plan objectives and processed with OsOne software. Features data were extracted from imaging data in OsOne software and exported to comma-separated values (CSV) files for further analysis.

\section{Infections}

Vero cells at $85-95 \%$ confluence in six-well plates were mock-infected or infected at a multiplicity of infection of 5 with $300 \mu$ of inoculum for $1 \mathrm{~h}$ and rocked every $15 \mathrm{~min}$ during the 1-h incubation. After $1 \mathrm{~h}, 2 \mathrm{ml}$ of fresh TC medium was added. Samples were imaged at 2, 4, 6, 8 and $10 \mathrm{~h}$ postinfection. For plaque assays, supernatant samples were taken and titrated.

\section{Data analysis}

For each condition (mock or infected at each time point), seven to ten images were taken 
at $10 \times$ magnification, merged in OsOne software and processed using the 'Generic Adherent' algorithm. Features' datasets were exported from OsOne software as CSV files and further analyzed in Microsoft Excel. Averages and standard deviations for the data points available were calculated and plotted. For each condition and time point, between 1239 and 2778 individual cells were analyzed. Sizes of analyzed populations are shown in Table 1. Representative individual phase and 3D intensity images were exported as Portable Network Graphics (.png) files from OsOne software.

\section{RESULTS \& DISCUSSION}

Healthy, growing Vero cells display an elongated morphology with clearly visible intracellular features, as shown in the example images in Figure 1, taken at 4x magnification. Next, we examined Vero cells at various time points postinfection to determine markers of infection that could be applied to rapid analytics. Visible signs of infection were detected by manual microscopic examination $24 \mathrm{~h}$ postinfection, which is in agreement with infection data that demonstrated cell rounding post-VSV infection due to the actions of the $M$

Figure 2. Time course of vesicular stomatitis virus-Lassa virus infection. Vero cells growing in six-well plates were either mock-infected or infected with VSV $\triangle$ G/LASVGP at a multiplicity of infection of 5 . At 2, 4, 6, 8 and 10 hp.i., seven to ten images of each mock-infected or infected wells were imaged at $10 \times$ magnification with a QMod camera. OsOne software was used for imaging and image processing. For each sample and time point, a representative image is shown in both phase-contrast and 3D optical intensity display modes. As the time course progresses, infected cells are starting to show signs of rounding up. The optical intensity images show increasing numbers of sharper peaks with jagged appearance. The bases of the peaks tend toward rounder shapes. In contrast, the mock cells retain their primary elongated shape.

VSV $\triangle$ G/LASVGP: Vesicular stomatitis virus expressing the Lassa virus Josiah glycoprotein. protein $[14,16]$. Additionally, VSV-infected cells have been demonstrated to show visible signs of infection less than $24 \mathrm{~h}$ postinfection and apoptosis in infected cells is induced very early in the infection process $[17,18]$. We performed time-course experiments with VSV $\Delta G / L A S V G P$ on Vero cells, collected supernatant samples and titered those (shown in Table 2), and imaged the cells using the Ovizio QMod camera and OsOne software. Uninfected cells display as smooth peaks of optical density, whereas infected cells show up as jagged peaks of varying optical density, indicating the apoptotic destruction of the infected cells. These changes become very apparent at $6 \mathrm{~h}$ postinfection. We also detected rounded cells near infected cells that did not display the jagged surface. Examination of Vero cells using the Ovizio system showed spots of high optical density for the nuclei and possibly mitochondria, as shown in Figure 2. Each cell had at least one spot of high optical density and a significant number of cells had two foci of high optical density. Infection with VSV $\Delta \mathrm{G}$ / LASVGP caused visible cell rounding and infected cells proceed to fragment and display roughness in 3D images, as shown in Figure 2.
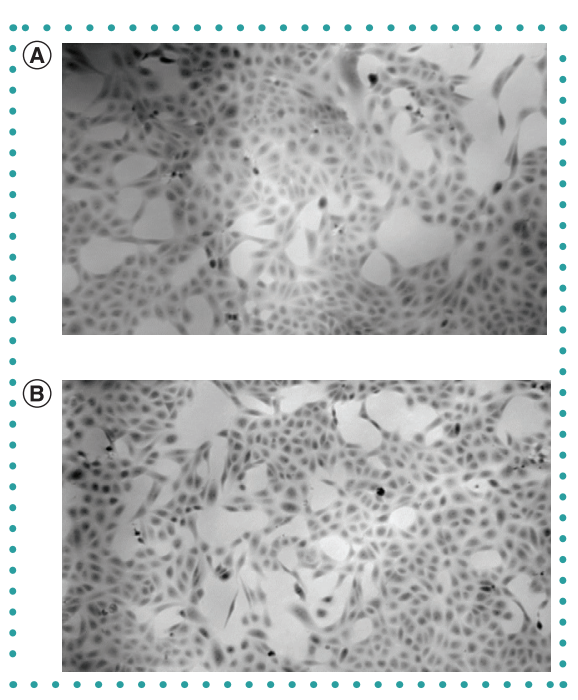

Figure 1. $4 \times$ magnified phase contrast images of Vero cells. Vero cells were grown in six-well culture dishes, imaged with a QMod camera using $4 \times$ magnification and exported. (A \& B) These images are representative of uninfected, healthy, subconfluent Vero cells, displaying elongated shapes.

OsOne software can be used to detect individual cells and measure a range of features on a per-cell basis. Collections of these measured features can then be extracted as CSV files and analyzed for

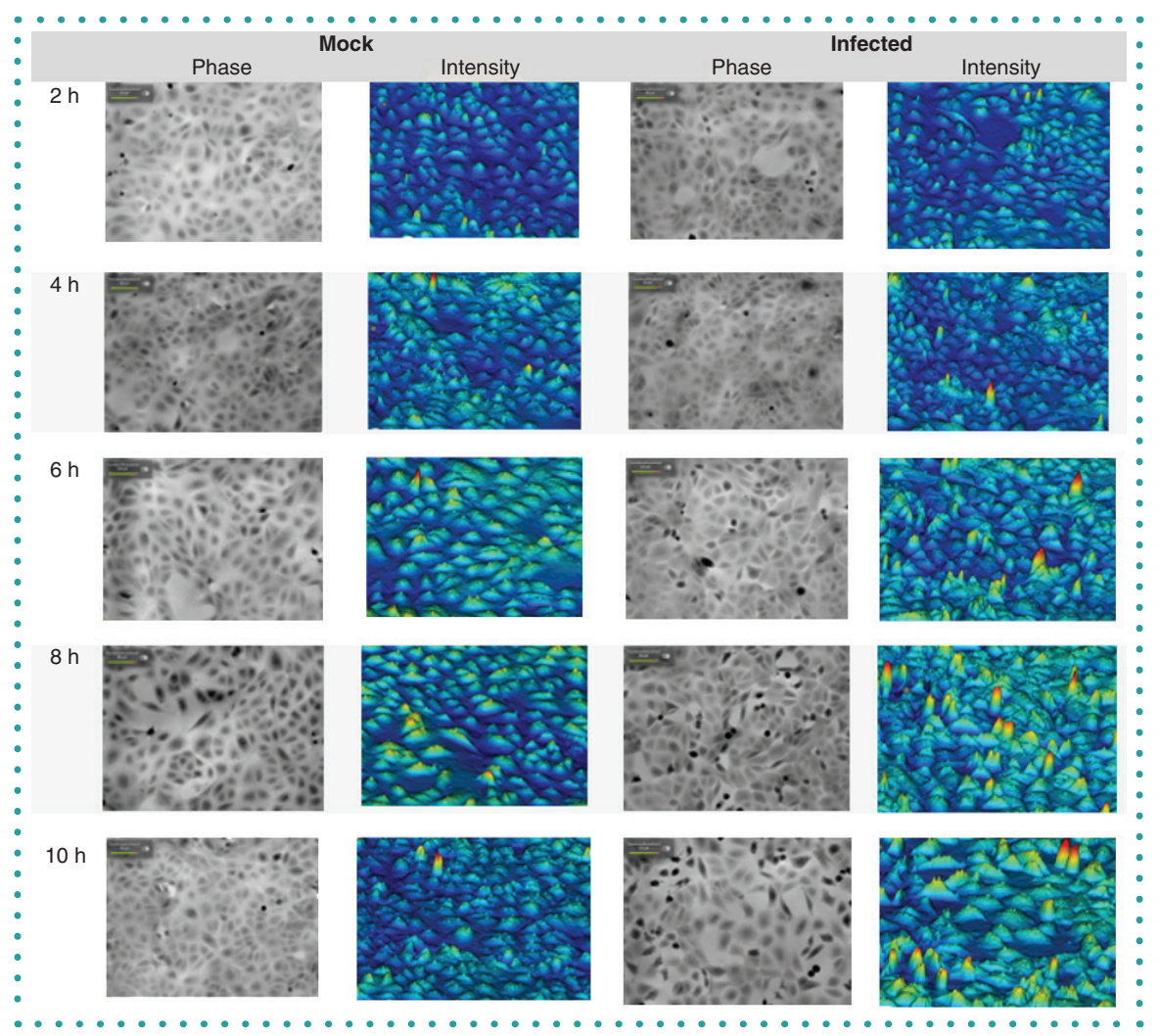



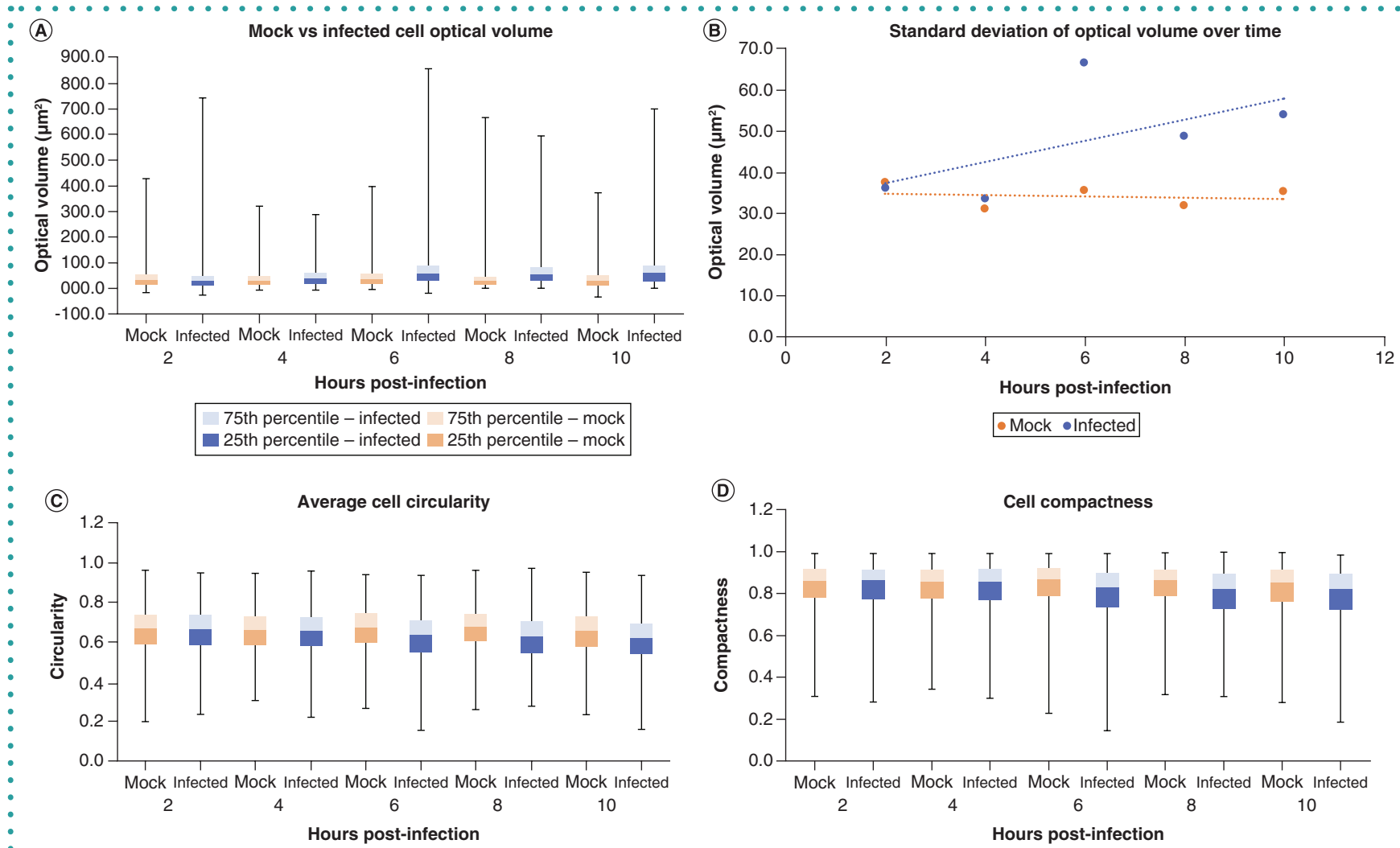

75th percentile - infected 75 th percentile - mock 25th percentile - infected 25 th percentile - mock

Figure 3. Selected cell features trends (continued on facing page). Features were measured on a per-cell basis in OsOne software. The distribution, central value and variability of the selected features were calculated from 1900 to 2200 per-cell measurements. Values were plotted as a function of time. (A) Optical volume shows no significant trend between VSV $\Delta$ G/LASVGP-infected cells and the mock-infected cells over the time course. (B) Standard deviation of optical volume also increases over time in VSV $\Delta$ G/LASVGP-infected cells, while remaining relatively constant or slightly declining in mock-infected cells. (C) In VSV $\Delta$ G/LASVGP-infected cells, average circularity declines over the time course and remains constant in mock-infected cells. (D) Compactness, as measured by the phase images, shows no significant difference between VSV $\Delta$ G/LASVGP-infected and mock-infected cells. (E) The optical height variance increases considerably in VSV $\Delta$ G/LASVGP-infected cells, while remaining constant in mock-infected cells. (F) The distribution of the mean intensity of VSV $\Delta$ G/LASVGP-infected cells shows a greater variance compared with the mock-infected cells. Additionally, a greater frequency of larger intensity values is shown in the VSV $\Delta$ G/LASVGP-infected cells. The graph was displayed as a strip plot to better illustrate this increasing frequency.

VSV $\Delta$ G/LASVGP: Vesicular stomatitis virus expressing the Lassa virus Josiah glycoprotein.

patterns. We examined measurements of individual cells and their changes over the time course of VSV $\Delta$ G/LASVGP infection. Box-andwhisker plots for each time point were plotted to represent the distribution and variability of our findings. Although our findings include an increase of the average optical volume, examining the variability of the volumes show that this increase is not significantly different from the findings in mock-infected cells at these time points (Figure $3 A$ \& B). It should be noted that we do see an increase in variability with VSV $\Delta G / L A S V G P$-infected cells as the time course progresses. Additionally, our captured images see an increasing number of cells increasing in circularity, software calculations yielded no apparent significant difference in circularity or compactness from 2 to $10 \mathrm{~h}$ postinfection (Figure $3 C \& \mathrm{D}$ ). However, it was determined that the optical height standard deviation increases with ongoing infection, although not exhibiting much change in uninfected cells (Figure $3 E$ ). Finally, we observed that the distribution of the intensity of VSV $\Delta \mathrm{G} /$ LASVGP-infected cells shows significantly greater standard deviation and increased frequency of higher intensity values, corresponding to the jagged peaks in the intensity images, compared with the mock-infected cells as seen in Figure 2. This trend can be observed as early as $4 \mathrm{~h}$ postinfection where captured images may be harder to distinguish (Figure 3F).

Considering the changes in cell morphology during VSV infection that can be visually observed, the variability of measured features show concordance. We examined features and found patterns that changed over the time course of VSV infection. Postinfection, the cell morphology changes; the cells tend to be round and the variability of the cell shape increases. It was determined that the microscope and software used have the ability to detect changes that are not yet visible to a human observer. Overall, the data demonstrate a method that can be used to monitor viral infection at early time points postinfection.

\section{FUTURE PERSPECTIVE}

Image analysis technologies continue to advance at a rapid pace. A large number of cellular parameters can be measurements on individual infected and uninfected cells in a 


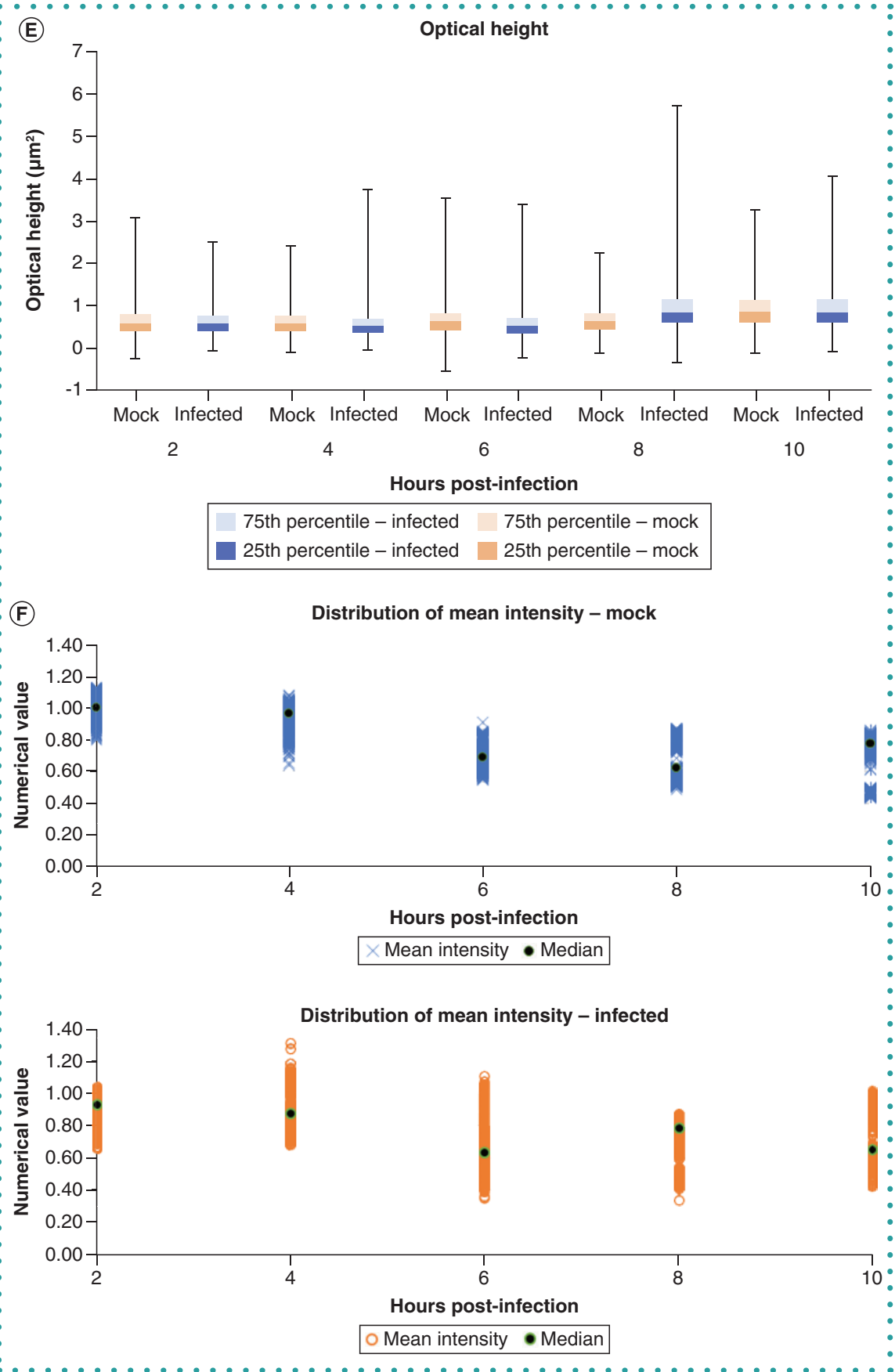

high-throughput fashion, which allows for the construction of predictive models. The features we examined showed patterns of cell morphology changes from 4 to $6 \mathrm{~h}$ postinfection. A standard VSV plaque assay requires $36-72 \mathrm{~h}$ for plaque formation and analysis, is time-consuming, uses many resources and is highly variable, whereas the microscopic method described here allows for earlier detection of infected cells in a rapid, highthroughput and less subjective manner. These factors are extremely important when analyzing the effects of antivirals or thera- peutics and determining virus production titers. We are continuing studies to examine features that are correlates of $V S V \Delta G$ / LASVGP infection in Vero cells and working toward building predictive models. The plaque assay is still the gold standard for viral titer determination; however, as mentioned earlier, although it is well established, the plaque assay is subject to high variability and is laborintensive. Novel methods that rely on operatorindependent measurements can reduce variability, resources and time required for performing such infectivity assays. An assay that images live cells without the need for dissociation or sample preparation, such as the techniques described, can yield significant time and resource savings. This method can also be adapted for other viruses by performing initial studies in a similar fashion and examining which measurable cell features change in response to infection. Other viruses are likely to show some differences in how they affect cell morphology, particularly if they are not lytic viruses.

Advances in image analysis, machine learning and computational power have brought significant progress toward fine examination of processes in cellular behavior. Basic mechanistic research, as well as process improvements in biologics manufacturing, will benefit immensely from the knowledge gained form the development of such assays.

\section{AUTHOR CONTRIBUTIONS}

I Scholz designed the study. E Vela supervised and advised the study. I Scholz and C Montoya performed the experiments. All authors analyzed the data, discussed the conclusions and prepared the manuscript.

\section{ACKNOWLEDGMENTS}

The authors would like to thank D Berrie, $S$ Doxilly, T Grow, A DeVries and the rest of the Process Development group at Ology Bioservices for support and discussions. We would also like to thank M Caple and R Cobb for manuscript reviews.

\section{FINANCIAL \& COMPETING INTERESTS DISCLOSURE}

The authors have no relevant affiliations or financial involvement with any organization or entity with a financial interest in or financial conflict with the subject matter or materials discussed in the manuscript. This includes employment, consultancies, honoraria, stock ownership or options, expert testimony, grants or patents received or pending, or royalties.

No writing assistance was utilized in the production of this manuscript.

\section{OPEN ACCESS}

This work is licensed under the AttributionNonCommercial-NoDerivatives 4.0 Unported License. To view a copy of this license, visit http://creativecommons.org/licenses/by-ncnd/4.0/ 


\section{REFERENCES}

Papers of special note have been highlighted as: • of interest; $\cdot$ • of considerable interest

1. Monath TP, Fast PE, Modjarrad K et al. rVSV $\triangle$ G-ZEBOVGP (also designated V920) recombinant vesicular stomatitis virus pseudotyped with Ebola Zaire glycoprotein standardized template with key considerations for a risk/benefit assessment. Vaccine 1, 100009 (2019).

2. Muik A, Dold C, Geiß Y et al. Semireplication-competent vesicular stomatitis virus as a novel platform for
oncolytic virotherapy. J. Mol. Med. (Berl.) 90(8), 959-970 (2012).

3. Velazquez-Salinas L, Naik S, Pauszek SJ, Peng KW, Russell SJ, Rodriguez LL. Oncolytic recombinant vesicular stomatitis virus (VSV) is nonpathogenic and nontransmissible in pigs, a natural host of VSV. Hum. Gene Ther. Clin. Dev. 28(2), 108-115 (2017)

4. Balachandran S, Porosnicu M, Barber GN. Oncolytic activity of vesicular stomatitis virus is effective against tumors exhibiting aberrant p53, Ras, or myc function and involves the induction of apoptosis. J. Virol. 75(7), 3474-3479 (2001).

5. Bishnoi S, Tiwari R, Gupta S, Byrareddy SN, Nayak D. Oncotargeting by vesicular stomatitis virus (VSV): advances in cancer therapy. Viruses 10(2), 90 (2018)

6. Melzer MK, Zeitlinger L, Mall S et al. Enhanced safety and efficacy of oncolytic VSV therapy by combination with $\mathrm{T}$ cell receptor transgenic T cells as carriers. Mol.
Ther. Oncolytics 12, 26-40 (2018)

7. Clarke DK, Hendry RM, Singh V et al. Live virus vaccines based on a vesicular stomatitis virus (VSV) backbone: standardized template with key considerations for a risk/benefit assessment. Vaccine 34(51), 6597-6609 (2016).

8. Stein DR, Warner BM, Soule $\mathrm{G}$ et al. A recombinant vesicular stomatitis-based Lassa fever vaccine elicits rapid and long-term protection from lethal Lassa virus infection in guinea pigs. NPJ Vaccines 4, 8 (2019).

9. Daddario-Dicaprio KM, Geisbert TW, Geisbert JB et al. Cross-protection against Marburg virus strains by using
a live, attenuated recombinant vaccine. J. Virol. $80(19)$, 9659-9666 (2006).

10. Mire CE, Geisbert JB, Agans KN et al. Use of single-injection recombinant vesicular stomatitis virus vaccine to protect nonhuman primates against lethal Nipah virus disease. Emerg. Infect. Dis. 25(6), 1144-1152 (2019).

11. Betancourt D, de Queiroz NM, Xia T, Ahn J, Barber GN. Cutting edge: innate immune augmenting vesicular stomatitis virus expressing Zika virus proteins confers protective immunity. J. Immunol. 198(8), 3023-3028 (2017).

12. Ding $X$, Liu N, Matsuo $K$, Sun $M$, Zhao X. Use of cell morphology as early bioindicator for viral infection. IET Nanobiotechnol. 8(1), 24-30 (2014).

13. Hebert CG, Dinardo N, Evans ZL, Hart SJ, Hachmann $A B$. Rapid quantification of vesicular stomatitis virus in Vero cells using laser force cytology. Vaccine 36(41)
6061-6069 (2018).

- Designed a very elegant and high-throughput method for infectivity analysis based on single-cell measurements.

14. Yakimovich A, Witte R, Andriasyan V, Georgi F, Greber UF. Label-free digital holo-tomographic microscopy reveals virus-induced cytopathic effects in live cells. mSphere 3(6), e00599-18 (2018).

- Established interesting methods based on changes in cell morphology to draw conclusions about infection state.

15. Ovizio Imaging Systems. 4D quantitative microscopy: real-time 3D imaging with differential digital holography microscopy (2019). http://www.ovizio.com/en/Technology

16. Gaddy DF, Lyles DS. Vesicular stomatitis viruses expressing wild-type or mutant $M$ proteins activate apoptosis through distinct pathways. J. Virol. 79(7), 4170-4179 (2005).

17. Gadaleta $P$, Vacotto $M$, Coulombié F. Vesicular stomatitis virus induces apoptosis at early stages in the viral cycle and does not depend on virus replication. Virus Res. 86(1-2), 87-92 (2002)

18. Felt SA, Grdzelishvili VZ. Recent advances in vesicular stomatitis virus-based oncolytic virotherapy: a 5-year update. J. Gen. Virol. 98(12), 2895-2911 (2017). 